JOURNAL OF THE

CHUNGCHEONG MATHEMATICAL SOCIETY

Volume 27, No. 1, 2014

http://dx.doi.org/10.14403/jcms.2014.27.1.133

\title{
A QUADRAPARAMETRIC FAMILY OF EIGHTH-ORDER ROOT-FINDING METHODS
}

\author{
YOUNG IK KIM*
}

\begin{abstract}
A new three-step quadraparametric family of eighthorder iterative methods free from second derivatives are proposed in this paper to find a simple root of a nonlinear equation. Convergence analysis as well as numerical experiments confirms the eighth-order convergence and asymptotic error constants.
\end{abstract}

\section{Introduction}

High-order iterative methods have been investigated by many researchers such as Bi-Ren-Wu[1], Bi-Wu-Ren[2], Chun-Ham[3], Kou-LiWang[6], Ren-Wu-Bi[7], Wang-Kou-Li[10] and Wang-Liu[11]. These methods have convergence order of at least 6 and are 3-step second derivativefree methods, the 2nd-step of which frequently uses King's fourth-order method[5], Jarratt's fourth-order method[4] or their variants. Undoubtedly, special attention has been paid to high-order iterative methods free from second derivatives to find a numerical solution of a nonlinear equation $f(x)=0$. In this paper, a three-step quadraparametric family of eighth-order methods free of second derivatives are proposed with their convergence results as well as numerical experiments for various test functions.

Let $f: \mathbb{C} \rightarrow \mathbb{C}$ have a simple root $\alpha$ and be analytic in a small region containing $\alpha$. A parametric family of three-step iterative methods are considered below: for $n=0,1, \cdots$,

Received December 27, 2013; Accepted January 16, 2014.

2010 Mathematics Subject Classification: Primary 65H05, 65 H99.

Key words and phrases: eighth-order convergence, efficiency index, quadraparametric family, asymptotic error constant.

*The author was supported by the research fund of Dankook University in 2013. 


$$
\left\{\begin{array}{l}
y_{n}=x_{n}-\frac{f\left(x_{n}\right)}{f^{\prime}\left(x_{n}\right)}, \\
z_{n}=y_{n}-K_{f}\left(x_{n}\right) \frac{f\left(y_{n}\right)}{f^{\prime}\left(x_{n}\right)}, \\
x_{n+1}=z_{n}-W_{f}\left(x_{n}\right) \frac{f\left(z_{n}\right)}{f^{\prime}\left(z_{n}\right)},
\end{array}\right.
$$

where, denoting $u_{n}=f\left(y_{n}\right) / f\left(x_{n}\right)$ and $v_{n}=f\left(z_{n}\right) / f\left(x_{n}\right)$,

$$
\begin{gathered}
K_{f}\left(x_{n}\right)=\frac{1+\beta u_{n}+\lambda u_{n}^{2}}{1+(\beta-2) u_{n}+\mu u_{n}^{2}}, \\
W_{f}\left(x_{n}\right)=\frac{1+a u_{n}+b v_{n}}{1+c u_{n}+d v_{n}},
\end{gathered}
$$

with $\beta, \lambda, \mu, a, b, c$ and $d$ as constant parameters to be determined later. We immediately find that both $K_{f}$ and $W_{f}$ are extensions proposed from classical King's method[5]. Functions $K_{f}$ and $W_{f}$ can be viewed as weighting functions for error terms $z_{n}-y_{n}$ and $x_{n+1}-z_{n}$, respectively. With a proper selection of the constant parameters based on the analysis to be presented in Section 2, they play a crucial role of maximizing the order of convergence up to 4 and 8 in the second step and third step. Observe that (1.1) requires 5 new function evaluations for $f\left(x_{n}\right), f\left(y_{n}\right), f\left(z_{n}\right), f^{\prime}\left(x_{n}\right)$ and $f^{\prime}\left(z_{n}\right)$ per iteration. We wish to reduce the number of function evaluations by one. To this end, we approximate $f^{\prime}\left(z_{n}\right)$ using $f\left(x_{n}\right), f\left(y_{n}\right), f\left(z_{n}\right), f^{\prime}\left(x_{n}\right)$. Taylor series expansion of $f^{\prime}\left(z_{n}\right)$ about $y_{n}$ leads to an approximation:

$$
f^{\prime}\left(z_{n}\right) \approx f^{\prime}\left(y_{n}\right)+f^{\prime \prime}\left(y_{n}\right)\left(z_{n}-y_{n}\right) .
$$

The fact that $y_{n} \approx z_{n}$ for sufficiently large values of $n$ yields the following approximations:

$$
\begin{gathered}
f^{\prime}\left(y_{n}\right) \approx \frac{f\left(z_{n}\right)-f\left(y_{n}\right)}{z_{n}-y_{n}}, \\
f^{\prime}\left(z_{n}\right) \approx \frac{f\left(x_{n}\right)-f\left(z_{n}\right)}{x_{n}-z_{n}}, \\
f^{\prime \prime}\left(y_{n}\right) \approx f^{\prime \prime}\left(z_{n}\right)=\frac{f^{\prime}\left(z_{n}\right)-f^{\prime}\left(x_{n}\right)}{z_{n}-x_{n}} \approx \frac{\frac{f\left(z_{n}\right)-f\left(x_{n}\right)}{z_{n}-x_{n}}-f^{\prime}\left(x_{n}\right)}{z_{n}-x_{n}} .
\end{gathered}
$$

Hence, $f^{\prime}\left(z_{n}\right)$ in (1.4) now approximates

$$
f^{\prime}\left(z_{n}\right) \approx \frac{f\left(z_{n}\right)-f\left(y_{n}\right)}{z_{n}-y_{n}}+\left(\frac{z_{n}-y_{n}}{z_{n}-x_{n}}\right)\left(\frac{f\left(z_{n}\right)-f\left(x_{n}\right)}{z_{n}-x_{n}}-f^{\prime}\left(x_{n}\right)\right) .
$$


Consequently, (1.1) can be rewritten as follows: with $K_{f}$ and $W_{f}$ described as in (1.2) and (1.3), respectively

$$
\left\{\begin{array}{l}
y_{n}=x_{n}-\frac{f\left(x_{n}\right)}{f^{\prime}\left(x_{n}\right)} \\
z_{n}=y_{n}-K_{f}\left(x_{n}\right) \frac{f\left(y_{n}\right)}{f^{\prime}\left(x_{n}\right)} \\
x_{n+1}=z_{n}-W_{f}\left(x_{n}\right) \frac{f\left(z_{n}\right)}{F\left(x_{n}\right)}
\end{array}\right.
$$

where

$$
F\left(x_{n}\right)=\frac{f\left(z_{n}\right)-f\left(y_{n}\right)}{z_{n}-y_{n}}+\left(\frac{z_{n}-y_{n}}{z_{n}-x_{n}}\right)\left(\frac{f\left(z_{n}\right)-f\left(x_{n}\right)}{z_{n}-x_{n}}-f^{\prime}\left(x_{n}\right)\right),
$$

explicitly depending on $f\left(x_{n}\right), f\left(y_{n}\right), f\left(z_{n}\right), f^{\prime}\left(x_{n}\right)$ not on $f^{\prime}\left(y_{n}\right)$.

Note that (1.9) now requires only four function evaluations for $f\left(x_{n}\right)$, $f\left(y_{n}\right), f\left(z_{n}\right), f^{\prime}\left(x_{n}\right)$ per iteration. The main objective of this paper is to show iteration scheme (1.9) has eighth-order convergence with relations

$$
\beta=\frac{1}{2}(\lambda-\mu-1), c=a, d=b-2,
$$

or equivalently with relations below:

$$
\begin{gathered}
K_{f}\left(x_{n}\right)=\frac{1+\frac{1}{2}(\lambda-\mu-1) u_{n}+\lambda u_{n}^{2}}{1+\frac{1}{2}(\lambda-\mu-5) u_{n}+\mu u_{n}^{2}}, \\
W_{f}\left(x_{n}\right)=\frac{1+a u_{n}+b v_{n}}{1+a u_{n}+(b-2) v_{n}},
\end{gathered}
$$

where $\lambda, \mu, a$ and $b$ are four independent constant parameters to be freely chosen in $\mathbb{R}$. In addition, deriving the asymptotic error constant or error equation is another goal of this paper. To measure convergence behavior within a given error bound, the values of $\left|x_{n}-\alpha\right|$ of proposed scheme (1.9) will be compared made with those of the existing seventhor eighth-order methods some of which are described as follows.

(1) Kou-Li-Wang[6]: seventh-order method

$$
\left\{\begin{array}{l}
y_{n}=x_{n}-\frac{f\left(x_{n}\right)}{f^{\prime}\left(x_{n}\right)}, \\
z_{n}=x_{n}-\frac{1+H_{2}\left(x_{n}, y_{n}\right)}{f^{\prime}\left(x_{n}\right)} \\
x_{n+1}=z_{n}-\left[\left(1+H_{2}\left(x_{n}, y_{n}\right)\right)^{2}+H_{\theta}\left(y_{n}, z_{n}\right)\right] \frac{f\left(z_{n}\right)}{f^{\prime}\left(z_{n}\right)}, \theta \in \mathbb{R}
\end{array}\right.
$$

with $H_{\theta}(y, z)=\frac{f(z)}{f(y)-\theta f(z)}$. The error equation of this method is asserted in [6] to satisfy the relation with $e_{n}=x_{n}-\alpha$ and $c_{j}=\frac{f^{(j)}(\alpha)}{j ! f^{\prime}(\alpha)}$ for $j=2,3,4$ :

$$
e_{n+1}=-2\left(c_{3}^{2}-2 c_{2}^{2} c_{3}+c_{2} c_{4}\right)\left(c_{2}^{2}-c_{3}\right) e_{n}^{7}+O\left(e_{n}^{8}\right) .
$$


REMARK 1.1. In fact, error equation (1.14a) asserted in the paper by $\mathrm{Kou}, \mathrm{Li}$ and Wang[6] has been found to be incorrect and needs to be completely corrected as the following:

$$
e_{n+1}=4 c_{2}^{2}\left(c_{2}^{2}-c_{3}\right)^{2} e_{n}{ }^{7}+O\left(e_{n}^{8}\right) .
$$

(2) Bi-Ren-Wu[1]: eighth-order method

$$
\left\{\begin{array}{l}
y_{n}=x_{n}-\frac{f\left(x_{n}\right)}{f^{\prime}\left(x_{n}\right)}, \\
z_{n}=y_{n}-\frac{2 f\left(x_{n}\right)-f\left(y_{n}\right)}{2 f\left(x_{n}\right)-5 f\left(y_{n}\right)} \frac{f\left(y_{n}\right)}{f^{\prime}\left(x_{n}\right)}, \\
x_{n+1}=z_{n}-\frac{f\left(x_{n}\right)+(2+\theta) f\left(z_{n}\right)}{f\left(x_{n}\right)+\theta f\left(z_{n}\right)} \cdot \frac{f\left(z_{n}\right)}{f\left[z_{n}, y_{n}\right]+f\left[z_{n}, x_{n}, x_{n}\right]\left(z_{n}-y_{n}\right)}, \theta \in \mathbb{R},
\end{array}\right.
$$

where $f[z, y]=\frac{f(z)-f(y)}{z-y}$ and $f[z, x, x]=\frac{f^{\prime}(x)-f[z, x]}{x-z}$. Observe that this method is a special case when $\lambda=\mu=0$ in (1.12) and $a=0, b=2+\theta$ in (1.13). The error equation of this method satisfies

$$
e_{n+1}=c_{2}{ }^{2} c_{3}\left(3 c_{2}{ }^{3}+2 c_{2} c_{3}-c_{4}\right) e_{n}{ }^{8}+O\left(e_{n}{ }^{9}\right) .
$$

(3) Bi-Wu-Ren[2]: eighth-order method

$$
\left\{\begin{array}{l}
y_{n}=x_{n}-\frac{f\left(x_{n}\right)}{f^{\prime}\left(x_{n}\right)}, \\
z_{n}=y_{n}-\left[\frac{f\left(x_{n}\right)}{f\left(x_{n}\right)-3 f\left(y_{n}\right)}\right]^{2 / 3} \cdot \frac{f\left(y_{n}\right)}{f^{\prime}\left(x_{n}\right)}, \\
x_{n+1}=z_{n}-\frac{f\left(x_{n}\right)+(2+\theta) f\left(z_{n}\right)}{f\left(x_{n}\right)+\theta f\left(z_{n}\right)} \cdot \frac{f\left(z_{n}\right)}{f\left[z_{n}, y_{n}\right]+f\left[z_{n}, x_{n}, x_{n}\right]\left(z_{n}-y_{n}\right)}, \theta \in \mathbb{R} .
\end{array}\right.
$$

The error equation of this method satisfies

$$
e_{n+1}=c_{2}{ }^{2} c_{3}\left(\frac{4}{3} c_{2}{ }^{3}+2 c_{2} c_{3}-c_{4}\right) e_{n}{ }^{8}+O\left(e_{n}{ }^{9}\right) .
$$

Some interesting choices of four parameters $(\lambda, \mu, a, b)$ in (1.12) and (1.13) will be further discussed in Section 2. Observe that (1.9) has four function evaluations per iteration and its efficiency index[9] is same as that of 3-step methods mentioned above. It's convergence order is optimal as well as consistent with the conjecture of Kung-Traub[8]. The advantage of proposed method (1.9) is that we can choose four free parameters as compared with methods (1.14), (1.15) and (1.16) having only one free parameter. Such a choice of four free parameters $(\lambda, \mu, a, b)$ gives a wide range of iterative methods which can be conveniently chosen from, depending on problems, to find a numerical root of the given nonlinear equation $f(x)$. Section 3 will discuss numerical experiments for various test functions. 


\section{Main results}

Development of proposed scheme (1.9) and its convergent analysis will be described in Theorem 2.1 below:

Theorem 2.1. Let $f$ and $\alpha$ be described as in Section 1. Let $c_{j}=$ $\frac{f^{(j)}(\alpha)}{j ! f^{\prime}(\alpha)}$ for $j=2,3, \cdots$. Assume that all three values $c_{2}, c_{3}$ and $c_{4}$ are not vanishing simultaneously. Let $x_{0}$ be an initial guess chosen in a sufficiently small neighborhood of $\alpha$. Let $\beta=\frac{1}{2}(\lambda-\mu-1), c=a$ and $d=b-2$ with $\lambda, \mu, a$ and $b$ as independent parameters freely chosen in $\mathbb{R}$. Then with $K_{f}\left(x_{n}\right)$ in (1.2) and $W_{f}\left(x_{n}\right)$ in (1.3), iteration scheme (1.9) defines a quadraparametric family of eighth-order methods and gives its error equation by

$$
e_{n+1}=c_{2}^{2} c_{3}\left\{2(a+1) c_{2} c_{3}-c_{4}+c_{2}^{3}(5 \lambda-\mu+3)\right\} e_{n}^{8}+O\left(e_{n}^{9}\right),
$$

where $e_{n}=x_{n}-\alpha$ for $n=0,1,2, \cdots$.

Proof. Taylor series expansion of $f\left(x_{n}\right)$ about $\alpha$ up to ninth-order terms yields with $f(\alpha)=0$ :

$$
\begin{gathered}
f\left(x_{n}\right)=f^{\prime}(\alpha)\left(e_{n}+c_{2} e_{n}^{2}+c_{3} e_{n}^{3}+c_{4} e_{n}^{4}+c_{5} e_{n}^{5}+c_{6} e_{n}^{6}\right. \\
\left.+c_{7} e_{n}^{7}+c_{8} e_{n}^{8}+c_{9} e_{n}^{9}+O\left(e_{n}^{10}\right)\right) .
\end{gathered}
$$

For ease of notation, $e_{n}$ will be denoted by $e$ (not to be confused with Napier's base for natural logarithms) for the time being. A lengthy algebraic computation induces relations (2.3)-(2.7) below:

$$
\begin{gathered}
f^{\prime}\left(x_{n}\right)=f^{\prime}(\alpha)\left(1+2 c_{2} e+3 c_{3} e^{2}+4 c_{4} e^{3}+5 c_{5} e^{4}+6 c_{6} e^{5}+7 c_{7} e^{6}\right. \\
\left.+8 c_{8} e^{7}+9 c_{9} e^{8}+O\left(e^{9}\right)\right), \\
\frac{f\left(x_{n}\right)}{f^{\prime}\left(x_{n}\right)}=e-c_{2} e^{2}+2\left(c_{2}^{2}-c_{3}\right) e^{3}+\left(-4 c_{2}^{3}+7 c_{2} c_{3}-3 c 4\right) e^{4}+ \\
\left(8 c_{2}^{4}-20 c_{2}^{2} c_{3}+6 c_{3}^{2}+10 c_{2} c_{4}-4 c_{5}\right) e^{5}+ \\
\left(-16 c_{2}^{5}+52 c_{2}^{3} c_{3}-33 c_{2} c_{3}^{2}-28 c_{2}^{2} c_{4}+17 c_{3} c_{4}+13 c_{2} c_{5}-5 c_{6}\right) e^{6}+ \\
2\left(16 c_{2}^{6}-64 c_{2}^{4} c_{3}-9 c_{3}^{3}+36 c_{2}^{3} c_{4}+6 c_{4}^{2}+9 c_{2}^{2}\left(7 c_{3}^{2}-2 c_{5}\right)+\right. \\
\left.11 c_{3} c_{5}+c_{2}\left(-46 c_{3} c_{4}+8 c_{6}\right)-3 c_{7}\right) e^{7}+\left(-64 c_{2}^{7}+304 c_{2}^{5} c_{3}-176 c_{2}^{4} c_{4}-\right. \\
75 c_{3}^{2} c_{4}+31 c_{4} c_{5}+c_{2}^{3}\left(-408 c_{3}^{2}+92 c_{5}\right)+4 c_{2}^{2}\left(87 c_{3} c_{4}-11 c_{6}\right)+27 c_{3} c_{6}+ \\
\left.c_{2}\left(135 c_{3}^{3}-64 c_{4}^{2}-118 c_{3} c_{5}+19 c_{7}\right)-7 c_{8}\right) e^{8}+O\left(e^{9}\right), \\
y_{n}=x_{n}-\frac{f\left(x_{n}\right)}{f^{\prime}\left(x_{n}\right)}=\alpha+c_{2} e^{2}-2\left(c_{2}^{2}-c_{3}\right) e^{3}+\left(4 c_{2}^{3}-7 c_{2} c_{3}+3 c 4\right) e^{4}-
\end{gathered}
$$




$$
\begin{aligned}
& 2\left(4 c_{2}^{4}-10 c_{2}^{2} c_{3}+3 c_{3}^{2}+5 c_{2} c_{4}-2 c_{5}\right) e^{5}+ \\
& \left(16 c_{2}^{5}-52 c_{2}^{3} c_{3}+33 c_{2} c_{3}^{2}+28 c_{2}^{2} c_{4}-17 c_{3} c_{4}-13 c_{2} c_{5}+5 c_{6}\right) e^{6}- \\
& 2\left(16 c_{2}^{6}-64 c_{2}^{4} c_{3}-9 c_{3}^{3}+36 c_{2}^{3} c_{4}+6 c_{4}^{2}+9 c_{2}^{2}\left(7 c_{3}^{2}-2 c_{5}\right)+\right. \\
& \left.11 c_{3} c_{5}+c_{2}\left(-46 c_{3} c_{4}+8 c_{6}\right)-3 c_{7}\right) e^{7}+\left(64 c_{2}^{7}-304 c_{2}^{5} c_{3}+176 c_{2}^{4} c_{4}+\right. \\
& 75 c_{3}^{2} c_{4}-31 c_{4} c_{5}+c_{2}^{3}\left(408 c_{3}^{2}-92 c_{5}\right)-4 c_{2}^{2}\left(87 c_{3} c_{4}-11 c_{6}\right)-27 c_{3} c_{6}- \\
& \left.c_{2}\left(135 c_{3}^{3}-64 c_{4}^{2}-118 c_{3} c_{5}+19 c_{7}\right)+7 c_{8}\right) e^{8}+O\left(e^{9}\right), \\
& f\left(y_{n}\right)=f^{\prime}(\alpha)\left(c_{2}^{2} e^{2}-2\left(c_{2}^{2}-c_{3}\right) e^{3}+\left(5 c_{2}^{3}-7 c_{2} c_{3}+3 c_{4}\right) e^{4}-\right. \\
& 2\left(6 c_{2}^{4}-12 c_{2}^{2} c_{3}+3 c_{3}^{2}+5 c_{2} c_{4}-2 c_{5}\right) e^{5}+ \\
& \left(28 c_{2}^{5}-73 c_{2}^{3} c_{3}+37 c_{2} c_{3}^{2}+34 c_{2}^{2} c_{4}-17 c_{3} c_{4}-13 c_{2} c_{5}+5 c_{6}\right) e^{6}- \\
& 2\left(32 c_{2}^{6}-103 c_{2}^{4} c_{3}-9 c_{3}^{3}+52 c_{2}^{3} c_{4}+6 c_{4}^{2}+c_{2}^{2}\left(80 c_{3}^{2}-22 c_{5}\right)+\right. \\
& \left.11 c_{3} c_{5}+c_{2}\left(-52 c_{3} c_{4}+8_{6}\right)-3 c_{7}\right) e^{7}+\left(144 c_{2}^{7}-552 c_{2}^{5} c_{3}+297 c_{2}^{4} c_{4}+\right. \\
& 75 c_{3}^{2} c_{4}+2 c_{2}^{3}\left(291 c_{3}^{2}-67 c_{5}\right)-31 c_{4} c_{5}-27 c_{3} c_{6}+c_{2}^{2}\left(-455 c_{3} c_{4}+54 c_{6}\right)+ \\
& \left.\left.c_{2}\left(-147 c_{3}^{3}+73 c_{4}^{2}+134 c_{3} c_{5}-19 c_{7}\right)+7 c_{8}\right) e^{8}+O\left(e^{9}\right)\right) . \\
& u_{n}=\frac{f\left(y_{n}\right)}{f\left(x_{n}\right)}=c_{2} e+\left(-3 c_{2}^{2}+2 c_{3}\right) e^{2}+\left(8 c_{2}^{3}-10 c_{2} c_{3}+3 c_{4}\right) e^{3}+ \\
& \left(-20 c_{2}^{4}+37 c_{2}^{2} c_{3}-8 c_{3}^{2}-14 c_{2} c_{4}+4 c_{5}\right) e^{4}+ \\
& \left(48 c_{2}^{5}-118 c_{2}^{3} c_{3}+55 c_{2} c_{3}^{2}+51 c_{2}^{2} c_{4}-22 c_{3} c_{4}-18 c_{2} c_{5}+5 c_{6}\right) e^{5}+ \\
& \left(-112 c_{2}^{6}+344 c_{2}^{4} c_{3}-252 c_{2}^{2} c_{3}^{2}+26 c_{3}^{3}-163 c_{2}^{3} c_{4}+\right. \\
& \left.150 c_{2} c_{3} c_{4}-15 c_{4}^{2}+65 c_{2}^{2} c_{5}-28 c_{3} c_{5}-22 c_{2} c_{6}+6 c_{7}\right) e^{6}+ \\
& \left(256 c_{2}^{7}-944 c_{2}^{5} c_{3}+480 c_{2}^{4} c_{4}+105 c_{3}^{2} c_{4}+c_{2}^{3}\left(952 c_{3}^{2}-207 c_{5}\right)-38 c_{4} c_{5}-\right. \\
& \left.34 c_{3} c_{6}+c_{2}^{2}\left(-693 c_{3} c_{4}+79 c_{6}\right)-2 c_{2}\left(114 c_{3}^{3}-51 c_{4}^{2}-95 c_{3} c_{5}+13 c_{7}\right)+7 c_{8}\right) e^{7}+ \\
& \left(-576 c_{2}^{8}+2480 c_{2}^{6} c_{3}-72 c_{3}^{4}-1336 c_{2}^{5} c_{4}+141 c_{3} c_{4}^{2}+\right. \\
& 132 c_{3}^{2} c_{5}-24 c_{5}^{2}+c_{2}^{4}\left(-3200 c_{3}^{2}+607 c_{5}\right)+c_{2}^{3}\left(2660 c_{3} c_{4}-251 c_{6}\right)- \\
& 46 c_{4} c_{6}-40 c_{3} c_{7}+3 c_{2}^{2}\left(418 c_{3}^{3}-159 c_{4}^{2}-292 c_{3} c_{5}+31 c_{7}\right)+ \\
& \left.c_{2}\left(-936 c_{3}^{2} c_{4}+258 c_{4} c_{5}+230 c_{3} c_{6}-30 c_{8}\right)+8 c_{9}\right) e^{8}+O\left(e^{7}\right) \text {. }
\end{aligned}
$$

Substituting relations (2.2)-(2.7) into (1.9), we get $x_{n+1}$ as follows by the aid of symbolic computation of Mathematica:

$$
x_{n+1}=\alpha+A_{5} e^{5}+A_{6} e^{6}+A_{7} e^{7}++A_{8} e^{8}+O\left(e^{9}\right),
$$

where $A_{i}=A_{i}(a, b, c, d, \beta, \lambda, \mu)(i=5,6,7,8,9)$ are multivariate polynomials in $a, b, c, d, \beta, \lambda$ and $\mu$; for instance,

$$
A_{5}=(c-a) c_{2}\left(-c_{2} c_{3}+c_{2}^{3}(1+2 \beta-\lambda+\mu)\right) .
$$


We impose conditions $A_{5}=A_{6}=A_{7}=0$ and $A_{8} \neq 0$ independently of $c_{j}$ 's so that iteration scheme (1.9) has eighth-order convergence. Solving $A_{5}=0$ for $c$ yields

$$
c=a .
$$

Substituting this $c$ into $A_{6}=0$ and $A_{7}=0$ after simplification yields

$$
c_{3}(-2+b-d)+c_{2}{ }^{2}(-b+d)(1+2 \beta-\lambda+\mu)=0,
$$

from which two relations follow independently of $c_{2}$ and $c_{3}$ :

$$
d=b-2, \beta=\frac{1}{2}(\lambda-\mu-1) .
$$

Substituting these $c, d, \beta$ into $A_{8}$ after simplification yields

$$
c_{2}^{2} c_{3}\left(2(1+a) c_{2} c_{3}-c_{4}+c_{2}^{3}(3+5 \lambda-\mu)\right) .
$$

Now restoring notation $e$ back to $e_{n}$ in (2.11) yields the error equation and the asymptotic error constant $\eta$ with convergence order 8 , respectively, as follows:

$$
\begin{aligned}
& e_{n+1}=c_{2}^{2} c_{3}\left\{2(a+1) c_{2} c_{3}-c_{4}+c_{2}^{3}(5 \lambda-\mu+3)\right\} e_{n}^{8}+O\left(e_{n}^{9}\right), \\
& \eta=\lim _{n \rightarrow \infty}\left|\frac{e_{n+1}}{e_{n}^{8}}\right|=\left|c_{2}^{2} c_{3}\left\{2(a+1) c_{2} c_{3}-c_{4}+c_{2}^{3}(5 \lambda-\mu+3)\right\}\right|,
\end{aligned}
$$

yielding desired (2.1). Substituting $d=b-2$ and $\beta=\frac{1}{2}(\lambda-\mu-1)$ found by (2.11) into (1.2) and (1.3) gives desired $K_{f}\left(x_{n}\right)$ in (1.12) and $W_{f}\left(x_{n}\right)$ in (1.13). This completes the proof.

Although iteration scheme (1.9) defines a quadraparametric family of eighth-order methods, it is interesting to observe that error equation (2.13) depends only on three parameters $\lambda, \mu, a$ and independent of parameter $b$. This kind of parameter-independency for the error equation occurs frequently as can be seen in many iterative methods such as (1.14), (1.15) and (1.16). Given choices of parameters, with the introduction of two normalized variables $u_{n}=f\left(y_{n}\right) / f\left(x_{n}\right)$ and $v_{n}=$ $f\left(z_{n}\right) / f\left(x_{n}\right)$, it is convenient to display a variety of $K_{f}\left(x_{n}\right), W_{f}\left(x_{n}\right)$ and $\eta$. It also simplifies coding of numerical Algorithm 3.1. Table 1 lists a number of such choices of $\lambda, \mu, a$ and $b$, being accompanied with $K_{f}\left(x_{n}\right), W_{f}\left(x_{n}\right)$ and $\eta$. 
TABle 1. Typical choices of $a, b, \lambda$ and $\mu$ for $K_{f}\left(x_{n}\right)$, $W_{f}\left(x_{n}\right)$ and $\eta$

\begin{tabular}{|c|c|c|c|c|}
\hline Case & $(\lambda, \mu, a, b)$ & $K_{f}\left(x_{n}\right)$ & $W_{f}\left(x_{n}\right)$ & $\eta$ \\
\hline 0 & $(0,0,0,3)$ & $\frac{2-u_{n}}{2-5 u_{n}}$ & $\frac{1+3 v_{n}}{1+v_{n}}$ & $\left|c_{2}^{2} c_{3}\left(3 c_{2}^{3}+2 c_{2} c_{3}-c_{4}\right)\right|$ \\
\hline 1 & $(-1,-2,-1,0)$ & $\frac{1-u_{n}^{2}}{1-2 u_{n}-2 u_{n}^{2}}$ & $\frac{1-u_{n}}{1-u_{n}-2 v_{n}}$ & $\left|c_{2}^{2} c_{3} c_{4}\right|$ \\
\hline 2 & $(-2,-7,-1,0)$ & $\frac{1+2 u_{n}-2 u_{n}^{2}}{1-7 u_{n}^{2}}$ & $\frac{1-u_{n}}{1-u_{n}-2 v_{n}}$ & $\left|c_{2}^{2} c_{3} c_{4}\right|$ \\
\hline 3 & $(0,-2,-1,0)$ & $\frac{2+u_{n}^{n}}{2-3 u_{n}-4 u_{n}^{2}}$ & $\frac{1-u_{n}}{1-u_{n}-2 v_{n}}$ & $\left|c_{2}^{2} c_{3}\left(5 c_{2}^{3}-c_{4}\right)\right|$ \\
\hline 4 & $\left(\frac{9}{16},-\frac{87}{16},-1,0\right)$ & $\frac{\left(4+9 u_{n}\right)\left(4+u_{n}\right)}{\left(16+8 u_{n}-87 u_{n}^{2}\right)}$ & $\frac{1-u_{n}}{1-u_{n}-2 v_{n}}$ & $\left|c_{2}^{2} c_{3}\left(\frac{45}{4} c_{2}^{3}-c_{4}\right)\right|$ \\
\hline 5 & $\left(\frac{9}{16}, \frac{73}{16},-1,0\right)$ & $\frac{\left(4-9 u_{n}\right)\left(4-u_{n}\right)}{\left(16-72 u_{n}+73 u_{n}^{2}\right)}$ & $\frac{1-u_{n}}{1-u_{n}-2 v_{n}}$ & $\left|c_{2}^{2} c_{3}\left(\frac{5}{4} c_{2}^{3}-c_{4}\right)\right|$ \\
\hline 6 & $\left(-\frac{9}{16}, \frac{39}{16},-1,0\right)$ & $\frac{\left(4-9 u_{n}\right)\left(4+u_{n}\right)}{\left(4-13 u_{n}\right)\left(4-3 u_{n}\right)}$ & $\frac{1-u_{n}}{1-u_{n}-2 v_{n}}$ & $\left|c_{2}{ }^{2} c_{3}\left(\frac{9}{4} c_{2}{ }^{3}+c_{4}\right)\right|$ \\
\hline 7 & $\left(-\frac{9}{16},-\frac{89}{16},-1,0\right)$ & $\frac{\left(4+9 u_{n}\right)\left(4-u_{n}\right)}{16-89 u_{n}^{2}}$ & $\frac{1-u_{n}}{1-u_{n}-2 v_{n}}$ & $\left|c_{2}^{2} c_{3}\left(\frac{23}{4} c_{2}^{3}-c_{4}\right)\right|$ \\
\hline 8 & $(1,4,-1,2)$ & $\left(\frac{1-u_{n}}{1-2 u_{n}}\right)^{2}$ & $\frac{1-u_{n}+2 v_{n}}{1-u_{n}}$ & $\left|c_{2}^{2} c_{3}\left(4 c_{2}^{3}-c_{4}\right)\right|$ \\
\hline 9 & $(0,-1,-1,2)$ & $\frac{1}{1-2 u_{n}-u_{n}^{2}}$ & $\frac{1-u_{n}+2 v_{n}}{1-u_{n}}$ & $\left|c_{2}^{2} c_{3}\left(4 c_{2}^{3}-c_{4}\right)\right|$ \\
\hline 10 & $(1,0,-1,1)$ & $\frac{1+u_{n}^{2}}{1-2 u_{n}}$ & $\frac{1-u_{n}+v_{n}}{1-u_{n}-v_{n}}$ & $\left|c_{2}^{2} c_{3}\left(8 c_{2}^{3}-c_{4}\right)\right|$ \\
\hline 11 & $(1,-4,-1,2)$ & $\frac{\left(1+u_{n}\right)^{2}}{1-4 u_{n}^{2}}$ & $\frac{1-u_{n}+2 v_{n}}{1-u_{n}}$ & $\left|c_{2}^{2} c_{3}\left(12 c_{2}^{3}-c_{4}\right)\right|$ \\
\hline 12 & $(2,1,-1,2)$ & $\frac{1+2 u_{n}^{2}}{\left(1-u_{n}\right)^{2}}$ & $\frac{1-u_{n}+2 v_{n}}{1-u_{n}}$ & $\left|c_{2}{ }^{2} c_{3}\left(12 c_{2}{ }^{3}-c_{4}\right)\right|$ \\
\hline 13 & $(1,-5,-1,2)$ & $\frac{\left(2+u_{n}\right)\left(1+2 u_{n}\right)}{\left(2+5 u_{n}\right)\left(1-2 u_{n}\right)}$ & $\frac{1-u_{n}+2 v_{n}}{1-u_{n}}$ & $\left|c_{2}^{2} c_{3}\left(13 c_{2}^{3}-c_{4}\right)\right|$ \\
\hline 14 & $(5,0,-1,1)$ & $1+2 u_{n}+5 u_{n}^{2}$ & $\frac{1-u_{n}+v_{n}}{1-u_{n}-v_{n}}$ & $\left|c_{2}{ }^{2} c_{3}\left(28 c_{2}{ }^{3}-c_{4}\right)\right|$ \\
\hline
\end{tabular}

\section{Algorithm, numerical results and discussions}

The analysis described in Section 2 allows us to develop a zero-finding algorithm to be implemented with Mathematica[12]:

Algorithm 3.1 (Zero-Finding Algorithm)

Step 1. Construct iteration scheme (1.1) with the given function $f$ having a simple zero $\alpha$ for $n \in \mathbb{N} \cup\{0\}$ as mentioned in Section 1 .

Step 2. Set the minimum number of precision digits. With exact or most accurate zero $\alpha$, supply the theoretical asymptotic error constant $\eta$, order of convergence $p$ as well as $c_{2}, c_{3}, c_{4}, \lambda$ and $\mu, a, b$ stated in Section 2. Set the error bound $\epsilon$, the maximum iteration number $n_{\max }$ and the initial guess $x_{0}$. Compute $\left|f\left(x_{0}\right)\right|$ and $\left|x_{0}-\alpha\right|$.

Step 3. Tabulate the values of $n, x_{n},\left|f\left(x_{n}\right)\right|,\left|e_{n}\right|=\left|x_{n}-\alpha\right|,\left|\frac{e_{n}}{e_{n-1}}\right|$ and $\eta$.

Throughout the numerical experiments, the minimum number of precision digits was chosen as 350, being large enough to minimize roundoff errors as well as to clearly observe the computed asymptotic error constants requiring small-number divisions. The zero $\alpha$, however, was separately computed with 700 digits of precision to have 400 significant digits, whenever its exact value is not known. The error bound $\epsilon=10^{-300}$ was used for moderately accurate computation. The values 
of initial guess $x_{0}$ were selected closely to $\alpha$ to guarantee the convergence. The computed asymptotic error constant agrees up to 8 significant digits with the theoretical one. The computed zero is accurate up to 300 significant digits, although the first 15 digits are displayed.

Iterative method YK1 with $(\lambda, \mu, a, b)=(-1,-2,-1,0)$ applied to test functions $f(x)=1-\frac{x}{2}+\sin ^{-1}\left(x^{2}-1\right), f(x)=e^{-x^{2}} \frac{\sin x}{x^{2}-1}+\cos x$. $\log (1+x-\pi)$ and $f(x)=e^{(x-1)^{2}+5}+(x-1)^{4}+5(x-1)^{2}-1$, clearly shows successful asymptotic error constants with eighth-order convergence for suitable initial values chosen near $\alpha$. Tables 2,3 and 4 list iteration indexes $n$, approximate zeros $x_{n}$, residual errors $\left|f\left(x_{n}\right)\right|$, errors $\left|e_{n}\right|=$ $\left|x_{n}-\alpha\right|$ and computational asymptotic error constants $\left|\frac{e_{n}}{e_{n-1}{ }^{8}}\right|$ as well as the theoretical asymptotic error constant $\eta$.

TABle 2. Convergence for

$f(x)=1-\frac{x}{2}+\sin ^{-1}\left(x^{2}-1\right)$ with $\alpha \approx 0.594810968398$

\begin{tabular}{|c||c|c|c|c|c|}
\hline$n$ & $x_{n}$ & $\left|f\left(x_{n}\right)\right|$ & $\left|e_{n}\right|=\left|x_{n}-\alpha\right|$ & $\left|\frac{e_{n}}{e_{n-1}}\right|$ & $\eta$ \\
\hline 0 & 0.7 & 0.114815 & 0.105189 & & \\
1 & 0.594810968314147 & $8.91737 \times 10^{-11}$ & $8.42220 \times 10^{-11}$ & 0.0056190363 & 0.0028544234 \\
2 & 0.594810968398369 & $7.65128 \times 10^{-84}$ & $7.22641 \times 10^{-84}$ & 0.0028544234 & \\
3 & 0.594810968398369 & $0 . \times 10^{-350}$ & $0 . \times 10^{-350}$ & & \\
\hline
\end{tabular}

TABLE 3. Convergence for

$f(x)=e^{-x^{2}} \frac{\sin x}{x^{2}-1}+\cos x \cdot \log (1+x-\pi)$ with $\alpha=\pi$

\begin{tabular}{|c||c|c|c|c|c|}
\hline$n$ & $x_{n}$ & $\left|f\left(x_{n}\right)\right|$ & $\left|e_{n}\right|=\left|x_{n}-\alpha\right|$ & $\left|\frac{e_{n}}{e_{n-1}}\right|$ & $\eta$ \\
\hline 0 & 2.965 & 0.191286 & 0.176593 & & \\
1 & 3.14159265248208 & $1.10772 \times 10^{-9}$ & $1.10771 \times 10^{-9}$ & 0.001171241754 & 0.000012094207 \\
2 & 3.14159265358979 & $2.74158 \times 10^{-77}$ & $2.74156 \times 10^{-77}$ & 0.000012094207 & \\
3 & 3.14159265358979 & $0 . \times 10^{-349}$ & $0 . \times 10^{-349}$ & & \\
\hline
\end{tabular}

TABLE 4. Convergence for

$f(x)=e^{(x-1)^{2}+5}+(x-1)^{4}+5(x-1)^{2}-1$ with $\alpha=1+i \sqrt{5}$

\begin{tabular}{|c||c|c|c|c|c|}
\hline$n$ & $x_{n}$ & $\left|f\left(x_{n}\right)\right|$ & $\left|e_{n}\right|=\left|x_{n}-\alpha\right|$ & $\left|\frac{e_{n}}{e_{n-1}}\right|$ & $\eta$ \\
\hline 0 & $0.96+2.3 i$ & 1.51612 & 0.0754142 & & \\
1 & $0.99999999909+2.23606797751 i$ & $1.622 \times 10^{-8}$ & $9.071 \times 10^{-10}$ & 0.86709637 & 0.13743472 \\
2 & $1.00000000000+2.23606797749 i$ & $1.127 \times 10^{-72}$ & $6.304 \times 10^{-74}$ & 0.13743472 & \\
3 & $1.00000000000+2.23606797749 i$ & $0 . \times 10^{-348}$ & $0 . \times 10^{-349}$ & & \\
\hline
\end{tabular}


TABLE 5. Comparison of $\left|x_{n}-\alpha\right|$ for various iterative methods

\begin{tabular}{|c|c|c|c|c|c|c|c|c|}
\hline$f(x)$ & $x_{0}$ & $\left|x_{n}-\alpha\right|$ & (KLW $)$ & $($ BRW $)$ & $\left(\begin{array}{c}\text { BWR } \\
a_{-1}\end{array}\right.$ & YK1 & YK5 & YK8 \\
\hline \multirow[t]{3}{*}{$f_{1}$} & \multirow[t]{3}{*}{-0.86} & $\left|x_{1}-\alpha\right|$ & $5.60 \mathrm{e}-07^{*}$ & $2.18 \mathrm{e}-07$ & $1.02 \mathrm{e}-07$ & $2.74 \mathrm{e}-08$ & $5.82 \mathrm{e}-08$ & $2.20 \mathrm{e}-07$ \\
\hline & & $\left|x_{2}-\alpha\right|$ & $1.03 \mathrm{e}-44$ & $2.38 \mathrm{e}-54$ & $3.37 \mathrm{e}-57$ & $2.31 \mathrm{e}-62$ & $2.67 \mathrm{e}-59$ & $2.71 \mathrm{e}-54$ \\
\hline & & $\left|x_{3}-\alpha\right|$ & $7.47 \mathrm{e}-309$ & $0 . e-350$ & $0 . e-350$ & $0 . e-350$ & 0.e-350 & 0.e-350 \\
\hline \multirow[t]{3}{*}{$f_{2}$} & \multirow[t]{3}{*}{1.45} & $\left|x_{1}-\alpha\right|$ & $1.00 \mathrm{e}-07$ & $2.41 \mathrm{e}-08$ & $1.82 \mathrm{e}-08$ & $6.14 \mathrm{e}-09$ & $1.02 \mathrm{e}-08$ & $2.21 \mathrm{e}-08$ \\
\hline & & $\left|x_{2}-\alpha\right|$ & $1.15 \mathrm{e}-49$ & $3.96 \mathrm{e}-61$ & $2.40 \mathrm{e}-62$ & $3.04 \mathrm{e}-67$ & $1.10 \mathrm{e}-64$ & $1.89 \mathrm{e}-61$ \\
\hline & & $\left|x_{3}-\alpha\right|$ & $3.05 \mathrm{e}-343$ & 0.e-349 & 0.e-349 & $0 . e-349$ & $0 . e-349$ & 0.e-349 \\
\hline \multirow[t]{3}{*}{$f_{3}$} & \multirow[t]{3}{*}{-1.3} & $\left|x_{1}-\alpha\right|$ & $4.95 \mathrm{e}-08$ & $3.29 \mathrm{e}-07$ & 2.51 & $6.22 \mathrm{e}-08$ & $1.00 \mathrm{e}-07$ & $2.71 \mathrm{e}-07$ \\
\hline & & $\left|x_{2}-\alpha\right|$ & $4.27 \mathrm{e}-52$ & $8.83 \mathrm{e}-51$ & $5.95 \mathrm{e}-52$ & $2.30 \mathrm{e}-57$ & $9.13 \mathrm{e}-56$ & $1.51 \mathrm{e}-51$ \\
\hline & & $\left|x_{3}-\alpha\right|$ & 0.e-349 & 0.e-349 & $0 . e-349$ & $0 . e-349$ & 0.e-349 & 0.e-349 \\
\hline \multirow[t]{4}{*}{$f_{4}$} & \multirow[t]{4}{*}{0.065} & $\left|x_{1}-\alpha\right|$ & $4.46 \mathrm{e}-07$ & $3.03 \mathrm{e}-09$ & $7.52 \mathrm{e}-11$ & $4.97 \mathrm{e}-10$ & $2.29 \mathrm{e}-10$ & $6.62 \mathrm{e}-09$ \\
\hline & & $\left|x_{2}-\alpha\right|$ & $7.60 \mathrm{e}-43$ & $2.49 \mathrm{e}-67$ & $1.70 \mathrm{e}-80$ & $2.49 \mathrm{e}-75$ & $1.08 \mathrm{e}-76$ & $1.60 \mathrm{e}-64$ \\
\hline & & $\left|x_{3}-\alpha\right|$ & $3.17 \mathrm{e}-293$ & 0.e-482 & $0 . e-508$ & $0 . e-498$ & 0.e-501 & $0 . \mathrm{e}-476$ \\
\hline & & $\left|x_{4}-\alpha\right|$ & & & & & & \\
\hline \multirow[t]{3}{*}{$f_{5}$} & \multirow[t]{3}{*}{-1.75} & $\left|x_{1}-\alpha\right|$ & $1.99 \mathrm{e}-07$ & $3.71 \mathrm{e}-08$ & $3.71 \mathrm{e}-08$ & $3.05 \mathrm{e}-08$ & $3.04 \mathrm{e}-08$ & $3.09 \mathrm{e}-08$ \\
\hline & & $\left|x_{2}-\alpha\right|$ & $3.25 \mathrm{e}-49$ & 2.61 & 2.51 & 6.6 & -63 & $8.03 e-63$ \\
\hline & & $\left|x_{3}-\alpha\right|$ & $9.95 \mathrm{e}-342$ & 0. & 19 & 49 & 49 & 0.e-349 \\
\hline \multirow[t]{3}{*}{$f_{6}$} & \multirow[t]{3}{*}{$1.65 i$} & $\left|x_{1}-\alpha\right|$ & $5.45 \mathrm{e}-08$ & $1.74 \mathrm{e}-08$ & $1.07 \mathrm{e}-08$ & 5.03 & $3.07 \mathrm{e}-08$ & $7.23 \mathrm{e}-09$ \\
\hline & & $\left|x_{2}-\alpha\right|$ & $3.17 \mathrm{e}-52$ & $3.72 \mathrm{e}-63$ & $2.28 \mathrm{e}-64$ & $1.42 \mathrm{e}-58$ & $2.23 \mathrm{e}-60$ & $9.94 \mathrm{e}-66$ \\
\hline & & $\left|x_{3}-\alpha\right|$ & 0.e-349 & 0.e-349 & 0.e-349 & $0 . e-349$ & 0.e-349 & 0.e-349 \\
\hline \multirow[t]{3}{*}{$f_{7}$} & \multirow[t]{3}{*}{1.3} & $\left|x_{1}-\alpha\right|$ & $2.12 \mathrm{e}-07$ & $5.85 \mathrm{e}-08$ & $4.68 \mathrm{e}-08$ & $8.68 \mathrm{e}-09$ & $1.95 \mathrm{e}-08$ & $3.51 \mathrm{e}-08$ \\
\hline & & $\left|x_{2}-\alpha\right|$ & $3.55 \mathrm{e}-4$ & 3.69 & 4.64 & $1.11 \mathrm{e}$ & $1.78 \mathrm{e}-62$ & $4.63 \mathrm{e}-60$ \\
\hline & & $\left|x_{3}-\alpha\right|$ & $1.29 \mathrm{e}-333$ & 0.e-349 & 0.e-349 & 0.e-349 & 0.e-349 & 0.e-349 \\
\hline
\end{tabular}

Convergence behavior was confirmed for further test functions below:

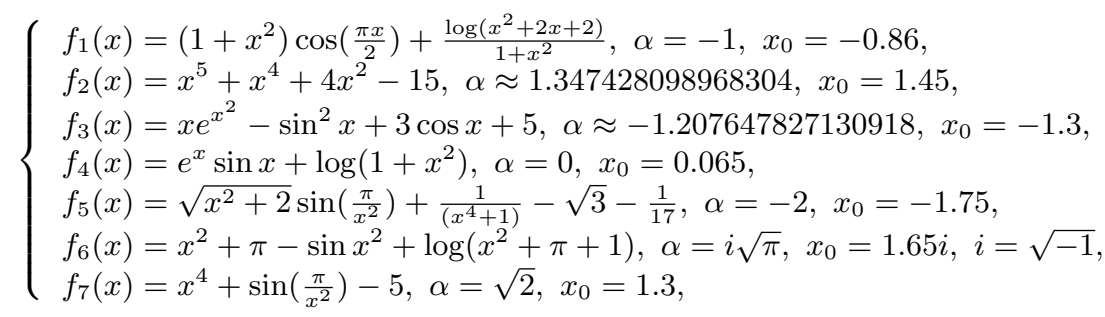

Here $\log z(z \in \mathbb{C})$ represents a principal analytic branch with $-\pi \leq$ $\operatorname{Im}(\log z)<\pi$.

Table 5 lists the values of $\left|x_{n}-\alpha\right|$ within the prescribed error bound for various seventh- or eighth-order methods KLW (1.14), BRW (1.15), BWR (1.16) and YK $i$ (1.9) identified by case number $i$ in Table 1.

As Table 5 suggests, proposed 3 -step methods $\mathbf{Y K} i$ show acceptable performance as compared with existing 3-step iterative methods KLW, BRW and BWR. Under the same order of convergence, one should note that the speed of local convergence of $\left|x_{n}-\alpha\right|$ is dependent on $c_{j}$, namely $f(x)$ and $\alpha$. During the current numerical experiments 
for the chosen test functions, YK1 has shown best performance for $f_{1}, f_{2}, f_{3}, f_{5}, f_{7}, \mathbf{Y K} 8$ for $f_{6}$, while $\mathbf{B W R}$ for $f_{4}$. The efficiency index defined by $E I=p^{1 / d}$, with $p$ as the order of convergence and $d$ the number of new evaluations of $f(x)$ or its derivatives per iteration, is equally $8^{1 / 4} \approx 1.68179$ for $\mathbf{Y K} i, \mathbf{B R W}$ and $\mathbf{B W R}$, and is better than $\sqrt{2}$, the efficiency index of Newton's method.

\section{References}

[1] W. Bi, H. Ren, and Q. Wu, Three-step iterative methods with eighth-order convergence for solving nonlinear equations, Journal of Computational and Applied Mathematics 225 (2009), 105-112.

[2] W. Bi, Q. Wu, H. Ren, A new family of eighth-order iterative methods for solving nonlinear equations, Applied Mathematics and Computation 214 (2009), 236-245.

[3] C. Chun and Y. Ham, Some sixth-order variants of Ostrowski root-finding methods, Applied Mathematics and Computation 193 (2007), 389-394.

[4] P. Jarratt, Some fourth-order multipoint iterative methods for solving equations, Math. Comput. 20 (1966), no. 95, 434-437.

[5] R. King, A family of fourth-order methods for nonlinear equations, SIAM J. Numer. Anal. 10 (1973), no. 5, 876-879.

[6] J. Kou, Y. Li, and X. Wang, Some variants of Ostrowski's method with seventhorder convergence, Journal of Computational and Applied Mathematics 209 (2007), 105-112.

[7] H. Ren, Q. Wu, and W. Bi, New variants of Jarratts method with sixth-order convergence, Numer. Algor. (2009), doi:10.1007/s11075-009-9302-3.

[8] H. T. Kung and J. F. Traub, Optimal order of one-point and multipoint iteration, Journal of the Association for Computing Machinery 21 (1974), 643-651.

[9] J. F. Traub, Iterative Methods for the Solution of Equations, Chelsea Publishing Company, 1982.

[10] X. Wang, J. Kou, Y. Li A variant of Jarratt method with sixth-order convergence, Applied Mathematics and Computation 204 (2008), 14-19.

[11] X. Wang, L. Liu Two new families of sixth-order methods for solving non-linear equations, Applied Mathematics and Computation 213 (2008), 73-78.

[12] S. Wolfram, The Mathematica Book, 4th ed., Cambridge University Press, 1999.

$*$

Department of Mathematics

Dankook University

Cheonan 330-714, Republic of Korea

E-mail: yikbell@dankook.ac.kr 